\title{
DETECTION OF INTESTINAL PROTOZOAN INFECTIONS WITH STRESS ON BLASTOCYSTIS, MICROSPORIDIA IN EGYPTIAN CHRONIC KIDNEY DISEASE PATIENTS
}

\section{By}

\author{
MONA HASSAN MOHAMMED ELSAYAD ${ }^{1 *}$, DALIA ALY MAHAREM ${ }^{2}$, FAIZA \\ AHMED SAEED ALI ${ }^{3}$ and NAGLAA FATHI ABD EL-LATIF ${ }^{* *}$
}

Departments of Parasitology ${ }^{1}$, and Internal Medicine ${ }^{2}$, Medical Research Institute ${ }^{1,2}$, Alexandria University, Egypt ' and Department of Hematology ${ }^{3}$, Republic Hospital, Mehweet, Yemen $\left({ }^{*}\right.$ Correspondence: Monaelsayad161@yahoo.com "dr_naglaafathi@hotmail.com; Tel.:+201224110570)

\section{Abstract}

This study assessed intestinal protozoa with stress on Blastocystis among patients with CKD and those on hemodialysis (HD) in Nephrology Unit, Medical Research Institute, Alexandria.

The patients were divided into three groups. GI: 50 patients on HD for at least one year, GII: $50 \mathrm{CKD}$ patients not on HD, and GIII: 50 healthy controls. Fresh stool samples were collected in labeled plastic boxes and examined as direct smears after staining with trichrome, Modified Ziehl-Neelsen (MZN) stains and modified trichrome (MT) stains. Besides, stool samples were cultured for Blastocystis hominis and Copro-antigen (ICT) was used to detect E. histolytica /dispar, G. lamblia and C. parvum.

The results showed that the parasitic infections were detected in $79 \%$ of the patients; $40 \%$ in HD and $39 \%$ in CKD patients. The positive samples were $(47 \%)$ detected by direct wet mount followed by iodine smear $(43 \%)$ and then trichrome stain (42\%). Microsporidiosis was detected in $55(55 \%) \mathrm{HD}$ and CKD patients using the MT stain. ICT agreed with microscopic examination in diagnosis of E.histolytica/ dispar and G. lamblia and showed perfect agreement with MZN in C. parvum diagnosis. Keywords: Chronic kidney disease patients, Intestinal protozoa.

\section{Introduction}

Intestinal protozoan infections (IPIs) constitute a significant problem worldwide with more than 58 million cases of IPIs each year, and more than $60 \%$ of the world's population being infected with at least one or more intestinal parasites during his/her life time. Numerous pathogenic protozoa inhabited the gastrointestinal tract of humans causing morbidity and mortality worldwide (Kucik et al, 2004). Several enteric protozoa were associated with diarrhea and debilitating sickness, especially in immunosuppressed persons (Fletcher et al, 2012). Species of Blastocystis, Cyclospora, Cryptosporidium, Dientamoeba, Entamoe$b a$, and Giardia were the commonest pathogenic ones (McHardy et al, 2014).

Despite the continuous improvement in the economic status, standard of living and the environmental sanitation of the Egyptian society, yet parasitic infections continue to be one of the most common public health problems (Mahfouz et al, 1997).

Infectious intestinal parasites are transmi- ted to humans through several ways, including contaminated food and water, inadequately treated sewage/sewage products, livestock and domestic pet handling. Foodborne transmission occurs during the harvesting, handling, and preparation processes, from cross-contamination with soiled implements, animal manure or contaminated water used for food preparation or via the food handlers themselves (Fletcher et al, 2012). Waterborne transmission still poses significant risks to human health both in developed and developing countries (Bridge et al, 2010). The cysts/ oocysts of several protozoa are highly resistant to conventional water treatment by chlorinetion (Carey et al, 2004). Immunodeficiency has deeply changed human-parasite relationships, and promoted the emergence or re-emergence of parasites, indeed, immune protection largely determines parasitic specificity and species barriers. Immune suppression weakens some inhibiting processes. Moreover, it may modify the host-parasite relationship and can promote the emer- 
gence of opportunistic infections (Lallo et al, 2012). Among immunocompromised group were patients suffering from chronic kidney disease (CKD), which once established, it causes progressive and irreversible loss of kidney function leading to the need for renal replacement therapy. Patients with end stage renal disease (ESRD) undergoing hemodialysis (HD) are individuals with significantly compromised immune system (Gil et al, 2013). So, the CKD was accepted as a gained immune deficiency. CKD has negative impacts on neutrophil chemotaxis, phagocytosis, and bactericidal actions as well as $\mathrm{T}$ cell function. The problems in maturing of the $\mathrm{T}$ lymphocytes increase susceptibility to infections (Meijers et al, 2012).

It is known that cellular immunity plays an important role in defense against parasitic diseases. Suppression of the immune system causes the increase of the pathogenic effects of the parasites and lead to the formation of severe clinical illnesses. CKD patients on HD had other combined conditions as diabetes, hypertension and $\mathrm{HCV}$ infection that increase the risk of infection. When the infection risks and related complications of these patients, who are vulnerable are considered, studies towards preventing infections are crucial. Also, it was important to study, factors that increase risk to opportunistic infections, such as Cryptosporidium, Cyclospora, Isospora belli, and Microsporidia, and other pathogenic intestinal parasites in patients with suppressed immunity (Stark et al 2009).

The work was aimed to detect intestinal protozoa with stress on Blastocystis sp., Microsporidia sp., using different diagnostic methods among CKD patients and those on HD in the Nephrology Unit, Medical Research Institute, Alexandria.

\section{Materials and methods}

A descriptive comparative case-control study was conducted on 150 subjects presenting to nephrology unit, Internal Medicine department and laboratory of parasit- ology department, Medical Research Institute (MRI). They were divided into three groups: GI: $50 \mathrm{HD}$ patients for at least one year, GII: 50 CKD patients not on HD, and GIII: 50 apparently healthy control. All did not have any anti-parasitic drugs one month prior to the study.

All participants were subjected to: Complete history taking including demographical data and clinical examination.

Stool examination. Fresh samples were divided into three portions: a- First portion for microscopic examination, samples were examined by direct wet saline smear; Formol ethyl acetate concentration method and Lugol's iodine smear (Garcia 2016). Permanent staining of fecal smears from concentrated fecal samples using: Trichrome stain, Modified trichrome stain (MTS), Modified Ziehl-Neelsen (Garcia, 2016), bSecond one for xenic cultivation of Blastocystis hominis (Jones, 1946), and c- Third one for copro-antigen detection for $E$. histolytica/dispar, G. lamblia \& C. parvum using Rida ${ }^{\circledR}$ Quick Entamoebal Giardial Cryptosporidium Combi Test (R-Biopharm AG, Germany) according to the manufactures' instructions.

The protocol of the study was approved by the Research Ethics Committee. All participants were volunteers in the study, after giving their informed written consent.

Statistical analysis: Data were fed to the computer and analyzed using IBM SPSS software package version 20.0. (Armonk, NY: IBM Corp). Qualitative data were described using number and percent. The Kolmogorov-Smirnov test was used to verify the normality of distribution. Quantitative data were described using range (minimum and maximum), mean, standard deviation and median. Significance of the obtained results was judged at the 5\% level. The results were considered significant if $\mathrm{p} \leq 0.05$.

\section{Results}

The mean age of patients in GI was $46.16 \pm 13.27$ years, but it was $43.56 \pm 12.66$ 
years in GII and $33.67 \pm 11.73$ years in GIII. Males constituted about $28 \%$ of GI \& 32 $\%$ of GII whereas $72 \%$ of GI \& $68 \%$ of G II were females, but in GIII, 52\% were males \& $48 \%$ were females. The overall parasitic infections was significantly higher in $\mathrm{HD}(80 \%)$ and $(78 \%)$ in CKD patients compared to the healthy controls $(10 \%)$. No helminthic parasites were found. Using direct wet mount, $B$. hominis was the most frequently detected protozoa in all groups. It was found in a significantly high in HD patients (46\%) and the CKD group (36\%) compared to control group (8\%). There was no significant difference between the studied groups regarding the percentage of $E$. histolyticaldispar and a non-pathogenic one Entamoeba coli. Giardia and Cryptosporidium sp. were detected in HD patients only ( $4 \%$ \& $2 \%$ respectively). While Microsporidia sp. was found in a significantly higher percentage of HD (48\%) and CKD (62\%) patients compared to the control group (Table 1). Single infection was detected in $65 \%$ of infected HD patients and $35 \%$ had double infection. While $71.8 \%$ of infected CKD patients had single infection and 28.2\% had double infection (Tab. 2).

The greatest percentage of protozoan positive samples $(47 \%)$ was detected by direct wet mount followed by Iodine smear in 43 cases $(43 \%)$ and Trichrome stain in 42 cases (42\%). Concerning B. hominis, direct smears and Iodine smears gave higher detection rates $(41 \%$ and $39 \%$ respectively) compared to Trichrome staining which missed 4 positive samples (37\%) but this difference didn't reach level of significance. As to the E. histolytica/dispar, direct smears and Iodine smears were superior to trichrome stain which missed one positive sample. Iodine smears missed two cases of $E$. coli, which were detected by both direct smears and Trichrome stain. Two G. lam- blia positive samples were diagnosed by the three parasitological methods (Tab. 3).

Out of 100 examined renal patients stool samples, only one case (1\%) had C. parvum infection using MZN stain in HD group. While 55 cases in the two groups HD and CKD (24 cases and 31 cases respectively) had Microsporidia infection using MTS (Tab. 4).

Microscopic examination of direct wet mount smears detected B. hominis in $41 \%$ patients (23 HD patients and $18 \mathrm{CKD}$ ) and in four apparently healthy controls. On the other hand xenic culture for $B$. hominis using Jones' medium detected only $38 \%$ of patients groups, missing three positive cases. Statistical analysis revealed very good agreement between direct smears and xenic culture method (Kappa index $=0.937^{*}$, $\mathrm{p}<0.001$ ) (Table 5).

G. lamblia was detected in two cases by ICT for copro-antigens, while E. histolytica and $C$. parvum were detected in only one case each (Tab. 6).

The two positive cases were diagnosed by microscopic examination by iodine and another one case was diagnosed by ICT, and, 97 cases were negative by both techniques. A moderate agreement was detected between both techniques in diagnosing $E$. histolytica (Tab. 7).

One positive case of $C$. parvum was diagnosed by both microscopic examination by MZN stain and ICT. Statistical analysis showed a Kappa index of 1 indicated the perfect agreement between the MZN stain and ICT in diagnosing C. parvum infection (Tab. 8).

There were two positive cases of $G$. lamblia detected by both the microscope and ICT. Statistical analysis showed a Kappa index of 1 indicated the perfect agreement between MZN stain and ICT in diagnosing G. lamblia infection as well (Tab. 9).

Table 1: Types of protozoan infection detected in groups

\begin{tabular}{|c|c|c|c|c|c|c|c|c|}
\hline Parasitic infection & \multicolumn{2}{|c|}{ G\I $(\mathrm{n}=50)$} & \multicolumn{2}{|c|}{ GII $(\mathrm{n}=50)$} & \multicolumn{2}{|c|}{ G\II $(\mathrm{n}=50)$} & \multirow{2}{*}{$\mathrm{P}$} \\
\cline { 2 - 6 } & No & $\%$ & No & $\%$ & No & $\%$ & & \\
\hline
\end{tabular}




\begin{tabular}{|c|c|c|c|c|c|c|c|c|}
\hline $\begin{array}{l}\text { Negative } \\
\text { Positive }\end{array}$ & $\begin{array}{l}10 \\
40 \\
\end{array}$ & $\begin{array}{l}20.0 \\
80.0 \\
\end{array}$ & $\begin{array}{l}11 \\
39 \\
\end{array}$ & $\begin{array}{l}22.0 \\
78.0 \\
\end{array}$ & $\begin{array}{c}45 \\
5 \\
\end{array}$ & $\begin{array}{l}90.0 \\
10.0 \\
\end{array}$ & $64.448 *$ & $<0.001 *$ \\
\hline Sig. bet. groups & \multicolumn{6}{|c|}{$\mathrm{P} 1=0.806, \mathrm{P} 2<0.001 *, \mathrm{P} 3<0.001 *$} & & \\
\hline \multicolumn{9}{|l|}{ Parasites detected } \\
\hline G. lamblia & 2 & 4.0 & 0 & 0.0 & 0 & 0.0 & 2.682 & $\mathrm{MCp}=0.329$ \\
\hline B. hominis & 23 & 46.0 & 18 & 36.0 & 4 & 8.0 & $18.476^{*}$ & $<0.001^{*}$ \\
\hline Sig. bet. Groups & \multicolumn{6}{|c|}{$\mathrm{P} 1=0.309, \mathrm{P} 2<0.001 *, \mathrm{P} 3=0.001 *$} & & \\
\hline $\begin{array}{c}\text { E. histolyti- } \\
\text { ca/dispar }\end{array}$ & 2 & 4.0 & 1 & \begin{tabular}{l|l|} 
& 2.0
\end{tabular} & 0 & 0.0 & 1.858 & $\mathrm{MCp}=0.780$ \\
\hline $\begin{array}{c}\text { Cryptosporidium } \\
\text { spp } \\
\end{array}$ & 1 & 2.0 & 0 & 0.0 & 0 & 0.0 & 1.831 & $\mathrm{MCp}=1.000$ \\
\hline E. coli & 2 & 4.0 & 0 & 0.0 & 1 & 2.0 & 1.858 & $\mathrm{MCp}=0.779$ \\
\hline Microsporidia spp & 24 & 48.0 & 31 & 62.0 & 0 & 0.0 & $45.531 *$ & $<0.001 *$ \\
\hline Sig. bet. Groups & \multicolumn{6}{|c|}{$\mathrm{P} 1=0.159, \mathrm{P} 2<0.001 *, \mathrm{P} 3<0.001 *$} & & \\
\hline
\end{tabular}

$\chi 2$ : Chi square test, MC: Monte CarloFE: Fisher Exact, $p$ : $p$ compared 3 groups, $p 1: p$ value for comparing between HD and CKD, p2: p compared between HD and Control, p3: $\mathrm{p}$ compared between CKD and Control, * significant at $\mathrm{p} \leq 0.05$ Table 2: Multiplicity of protozoal infection among infected subjects

\begin{tabular}{|c|c|c|c|c|c|c|c|c|}
\hline \multirow{2}{*}{ Infection status } & \multicolumn{2}{|c|}{ GI $(\mathrm{n}=40)$} & \multicolumn{2}{|c|}{ GII $(\mathrm{n}=39)$} & \multicolumn{2}{|c|}{ GIII $(\mathrm{n}=5)$} & \multirow{2}{*}{ MCp } \\
\cline { 2 - 7 } & No & $\%$ & No & $\%$ & No & $\%$ & & \\
\hline Single & 26 & 65 & 28 & 71.8 & 5 & 100 & 2.282 & 0.351 \\
Double & 14 & 35 & 11 & 28.2 & 0 & 0.0 & & \\
\hline
\end{tabular}

Table 3: Protozoal infection by different techniques among renal patients

\begin{tabular}{|c|c|c|c|c|c|c|c|c|}
\hline \multirow[t]{2}{*}{ Types of parasite } & \multicolumn{2}{|c|}{ Direct smear } & \multicolumn{2}{|c|}{ Iodine smear } & \multicolumn{2}{|c|}{ Trichome stain } & \multirow[b]{2}{*}{$\chi^{2}$} & \multirow[b]{2}{*}{$\mathrm{P}$} \\
\hline & No & $\%$ & No & $\%$ & No & $\%$ & & \\
\hline B. hominis & 41 & 41.0 & 39 & 39.0 & 37 & 37.0 & 0.336 & 0.845 \\
\hline Sig. bet. & \multicolumn{6}{|c|}{$\mathrm{P} 1=0.773, \mathrm{P} 2=0.562, \mathrm{P} 3=0.771$} & & \\
\hline E.histolytica/dispar & 2 & 2.0 & 2 & 2.0 & 1 & 1.0 & 0.592 & $\mathrm{MCp}=1.000$ \\
\hline Sig. bet. & \multicolumn{6}{|c|}{ FEp1 $=1.000$, FEp $2=1.000$, FEp $3=1.000$} & & \\
\hline E. coli & 2 & 2.0 & 0 & 0.0 & 2 & 2.0 & 2.080 & $\mathrm{MCp}=0.553$ \\
\hline Sig. bet. & \multicolumn{6}{|c|}{ FEp1 $=0.497$, FEp2 $=1.000$, FEp3 $=0.497$} & & \\
\hline G. lamblia & 2 & 2.0 & 2 & 2.0 & 2 & 2.0 & 0.220 & $\mathrm{MCp}=1.000$ \\
\hline Sig. bet. & \multicolumn{6}{|c|}{ FEp $1=1.000$, FEp $2=1.000, F E p 3=1.000$} & & \\
\hline
\end{tabular}

Table 4: Detection of Cryptosporidium sp. and Microsporida sp. among renal patients using special stains

\begin{tabular}{|c|c|c|}
\hline \multirow{2}{*}{ Special stain } & \multicolumn{2}{|c|}{ Positive } \\
\cline { 2 - 3 } & No & $\%$ \\
\hline Cryptosporidium sp. (M.Z.N) & 1 & 1.0 \\
\hline Microsporidia sp. (M.T.S) & 55 & 55.0 \\
\hline
\end{tabular}

Table (5): Agreement analysis of B. hominis detected by direct smear and xenic Culture method

\begin{tabular}{|c|c|c|c|c|c|c|}
\hline Techniques & \multicolumn{3}{|c|}{ Direct smear } & \multicolumn{2}{|c|}{ Xenic culture } & \multirow{2}{*}{$\mathrm{P}$} \\
\cline { 2 - 5 } B. hominis & No & $\%$ & No & $\%$ & & \\
\hline Negative & 59 & 59.0 & 62 & 62.0 & $88.198^{*}$ & $<0.001^{*}$ \\
Positive & 41 & 41.0 & 38 & 38.0 & & \\
\hline Total & 100 & 100.0 & 100 & 100.0 & & \\
\hline Kappa & \multicolumn{7}{|c|}{0.937 (very good) } & & \\
\hline
\end{tabular}

Table 6: Distribution of protozoa infection among 100 individuals tested by ICT

\begin{tabular}{|c|c|c|}
\hline \multirow{2}{*}{} & \multicolumn{2}{|c|}{ Positive } \\
\cline { 2 - 3 } & No & $\%$ \\
\hline E. histolytica/dispar & 1 & 1.0 \\
Giardia lamblia & 2 & 2.0 \\
Cryptosporidium $\mathrm{sp}$. & 1 & 1.0 \\
\hline
\end{tabular}

ICT: Immuno-chromatographic test

Table 7: Agreement between microscopic examination (Iodine) and ICT for E. histolytica/dispar

\begin{tabular}{|c|c|c|c|}
\hline \multirow{2}{*}{ Microscopic examination (Iodine) } & \multicolumn{2}{|c|}{ Immuno-chromatographic test (ICT) } & \multirow{2}{*}{ Total } \\
\cline { 2 - 4 } & Positive & Negative & 2 \\
Positive & 0 & 2 & 98 \\
\hline Negative & 1 & 97 & 1 \\
\hline Total & 1 & 99 & \\
\hline
\end{tabular}

Table 8: Agreement between microscopic examination by MZN stain and ICT for Cryptosporidium sp.

M.Z.N stain

\begin{tabular}{|l|l|l|}
\hline \multicolumn{2}{|c|}{ ICT } & \multirow{2}{*}{ Total } \\
\hline Positive & Negative & Tot \\
\hline
\end{tabular}




\begin{tabular}{|l|c|c|c|}
\hline Positive & 1 & 0 & 1 \\
Negative & 0 & 99 & 99 \\
\hline Total & 1 & 99 & 100 \\
\hline
\end{tabular}

Kappa index $=1, \mathrm{p}<0.001$ perfect agreement

Table (9): Agreement between microscopic technique and ICT for detection of G. lamblia

\begin{tabular}{|l|c|c|c|}
\hline \multirow{2}{*}{ Microscope } & \multicolumn{2}{|c|}{ ICT } & Total \\
\cline { 2 - 4 } \multicolumn{1}{|c|}{ Positive } & Negative & 2 \\
Positive & 2 & 0 & 98 \\
Negative & 0 & 98 & 100 \\
\hline Total & 2 & 98 & 0 \\
\hline
\end{tabular}

\section{Discussion}

Protozoa diseases were considered major causes of morbidity and mortality in the developing world. The segment of the population with significant defects in the immune system continues to grow. Chronic diseases that include asplenia, CKD and chronic hepatic disease are considered as immunosuppression status. These patients catch parasitic infections more easily (Abdel-Hafeez et al, 2012, Kamki et al, 2015, Bora et al, 2016).

$B$. hominis is an obligate anaerobic protozoan found in human and animal's large intestine, and is the most common eukaryotic organism reported in human fecal samples (Zierdt et al, 1967). Its diagnose depended on laboratory techniques such as routine direct microscopy of wet preparations or stained with trichrome or iodine stain, the cultivation and immuno-assay methods (Elghareeb et al, 2015). Cryptosporidium, Isospora belli, and microsporidia are intestinal protozoa that cause obligatory intracellular infections. They are transmitted either by stool from person to person or through contaminated water or food by spores or oocysts (Goodgame, 1996).

In the present work, the majority of participants were older than 40 years $(66 \%$ GI \& $68 \%$ GII) and about two thirds of them were females $(70 \%)$. The overall percentage of protozoa detected in patients was $79 \%\{\mathrm{HD}(40 \%)$ and CKD patients (39\%) $\}$ in the following frequency:55 \% Microsporidia spp, $41 \%$ B. hominis, 3\% E. histolytica, $2 \%$ for each of the G. lamblia and $E$. coli and 1\% Cryptosporidium spp., without helminthes. Of 79 infected patients, single protozoan was in 54 patients $(68 \%)$ and double protozoa were in $25(31.6 \%)$. Regarding controls; four individuals were infected with $B$. hominis and one with the non-pathogenic $E$. coli. The results agreed with Shehata et al (2019) who found a significantly higher prevalence rate of intestinal parasitoses among HD patients compared to apparently healthy controls (52.5\% vs. $12.0 \%$, respectively), and without helminthes. The parasites among patients were Cryptosporidium sp. (32.5\%), B. hominis $(24.2 \%)$ and microsporidia (11.7\%). Ali et al. (2000) in HD patients and controls with diarrhea detected $33.3 \%$ protozoa in patients \& $5 \%$ in controls. C. parvum was found in 15\% and Microsporidia in $8.3 \%$ of patients. Kulik et al. (2008) reported that Blastocystis sp. in $21 \%$ of HD patients, in mixed or single infections, while Cryptosporidium sp. and $E$. coli were $4.7 \%$ for each one. The difference in prevalence may be due to differences in population demographics, behavior, nutritional status, educational level, socioeconomic and seasonal factors, as well as diagnostic methods (AlHindi et al, 2008).

In the present study, some protozoa positive samples were missed by trichrome staining. The positive samples $(47 \%)$ were detected by direct wet mount followed by iodine smear $(43 \%)$ and then trichrome stain $(42 \%)$. It was hypothesized that wet mount in physiological saline always been the mainstay of any initial laboratory examination to detect motile trophozoites, but difficult to distinguish non-motile amoebae from macrophages or polymorphonuclear leucocytes. Also, the larger and more ma- 
ture cyst forms of E. coli and E. histolytica were not well stained by trichrome but detected by concentration (Shetty et al, 1988). However, Shoaib et al. (2002) found that Trichrome stain of fecal smears was better in diagnosis of protozoa as compared to the conventional wet mount. They added that morphological organism features were noticeable against the pale background making the visibility of protozoa more prominent and increased its sensitivity. Darabian et al. (2016) showed that trichrome staining was more sensitive than both direct smear and formalin ethyl acetate sedimentation techniques in the detection of $B$. hominis in stool samples of patients with the irritable bowel syndrome (IBS). However, an obvious disadvantage is the tedious protocol of Trichrome staining which requiresd an hour fixation and a total time of about two hours to complete. Also, appropriate fixation periods coupled with sufficient washing steps are significant in obtaining a well-stained nucleus; this may require the preparation of a number of slides for each stool sample. Repeated use of acid alcohol in de-staining trichrome stain decreased its efficiency and required a longer time although a better alternative was fresh solution and needed skills of an experienced microscopist (Tan et al, 2010).

In the present study, microsporidiosis was in 55 cases $(55 \%)$ in both HD \& CKD, by modified Trichrome stain. El-Nadi et al. (2004) reported that fecal samples from HD patients and cultured on modified agar plate, $\&$ subjected to formol-ether and sucrose sugar concentration method then MZN acid-fast staining, showed C. parvum, C. cayetanensis, Isospora belli and Microsporidia in $48 \%, 12 \%, 4 \%$ and $2 \%$ respectively.

Light microscopy proved to be simple, easy, least expensive method to detect the Blastocypstis spp. in feces (Garcia, 2016).

The present study showed an agreement between direct smears and xenic culture method for B. hominis. Kukoschke et al. (1990) did not find any difference between microscopy and cultures methods. They used a highly nutritive biphasic medium for isolation, causing an overgrowth of bacteria, resulting in low isolation rate of Blastocystis. But, culture may allow preferential growth of specific strains while eliminating others false negative cases. The Jones' medium proved good for xenic culture of Blastocystis spp. and several factors affected detection of Blastocystis spp. via culture method and various available media (Leelayoova et al, 2002; Stensvold et al, 2007).

Termmathurapoj et al (2004) found that in vitro cultivation was the 'gold standard' for B. hominis. Yakoob et al. (2010) reported that $B$. hominis was positive by stool microscopy in $49 \%$, by culture in $53 \%$ and by PCR positive in $44 \%$ of cases.

Since multiple protozoa infections coexised in the same sample, there was a need for improved diagnostic procedures, as rapid immunoassay test for E. histolytica/ dispar, Giardia lamblia and Cryptosporidium spp. The triage parasite panel was a qualitative stool enzyme immunoassay capable of detecting any of these parasites in fresh or frozen human fecal specimens (Goni et al, 2012). In the present results, triple ICT copro-antigen were as followed for E. histolytica antigen detected in $1 \%$ of cases, $G$. lamblia in $2 \%$, while Cryptosporidium antigen in $1 \%$. There was an agreement between ICT and the microscopic technique stressed on \reliability to diagnose G. lamblia and C. parvum and a moderate agreement was between ICT and microscopic technique to diagnose E. histolytica. Swierczewski et al. (2012) compared the results of 266 stool samples to the triage parasites panel, sensitivity and specificity for $E$. histolytica/dispar were $100 \%$ \& $100 \%$, for $G$. lamblia: $100 \%$ \& $100 \%$ and C. parvum: $70 \%$ \& $100 \%$, without cross reactivity with other stool parasites. This agreed with Garcia et al. (2000).

Goni et al. (2012) considered microscopy as gold standard and PCR as reference technique to differentiate between E. histo- 
lytica and $E$. dispar, although both have limitations, agreement with microscopy and PCR was over $90 \%$, but agreement between microscopy and ICT for E. histolytica was $76.3 \%$, due to the microscopic inability to differentiate $E$. histolytica from nonpathogenic E. dispar (Goni et al, 2012). The ICT advantages were being simple, rapid, completed in less than 15 minutes, used with either fresh or frozen unfixed stool, easily read, and interpreted as compared to ova examination, without cross reactivity with other intestinal parasites (coated with monoclonal antibodies).

\section{Conclusion}

Microsporidia was the most common protozoa detected among renal patients followed by $B$. hominis. ICT can replace the staining methods in diagnosis of $E$. histolytica/dispar, G. lamblia, and C. parvum.

\section{References}

Abdel-Hafeez, EH, Ahmad, AK, Ali, BA, Moslam, FA, 2012: Opportunistic parasites among immune-suppressed children in Minia District, Egypt. Korean J. Parasitol. 50, 1:5762.

Al-Hindi, AI, El-Kichaoi, A, 2008: Occurrence of gastrointestinal parasites among preschool children, Gaza, Palestine. Islamic Univ. J. 16:125-30.

Ali, MS, Mahmoud, LA, Abaza, BE, Ramadan, MA, 2000: Intestinal spore-forming protozoa among patients suffering from chronic renal failure. J. Egypt. Soc. Parasitol. 30, 1:93100.

Bora, I, Dutta, V, Lyngdoh, WV, Khyriem, AB, Durairaj, E, et al, 2016: Study of intestinal parasites among the immunosuppressed patients attending a tertiary-care center in Northeast India. Int. J. Med. Sci. Publ. Hlth. 5: 924-9. Bridge, JW, Oliver, DM, Chadwick, D, Godfray, HC, Heathwaite, AL, et al, 2010: Engaging with the water sector for public health benefits: waterborne pathogens and diseases in developed countries. Bull. WHO 88, 11:873-5. Carey, CM, Lee, H, Trevors, JT, 2004: Biology, persistence and detection of Cryptosporidium parvum and Cryptosporidium hominis oocyst. Water Res. 38, 4:818-62.

Darabian, A, Berenji, F, Ganji, A, Fata, A, Jarahi, L, 2016: Association between Blasto- cystis hominis and irritable bowel syndrome (IBS). Hlth. Sci. 59:102-5.

Elghareeb, AS, Younis, MS, EI Fakahany, AF, Nagaty, IM, Nagib, MM, 2015: Laboratory diagnosis of Blastocystis spp. in diarrheic patients. Trop. Parasitol. 5, 1:36-41.

El-Nadi, NA, Taha, A, 2004: intestinal parasites detected among haemodialysis patients in Sohag University Hospital El-Minia. Med. Bull. 15, 2:233-9.

Fletcher, SM, Stark, D, Harkness, J, Ellis, J, 2012: Enteric protozoa in the developed world: a public health perspective. Clin. Microbiol. Rev. 25, 3:420-49.

Garcia, LS, Shimizu, RY, Bernard, CN, 2000: Detection of Giardia lamblia, Entamoeba histolytica/Entamoeba dispar, and Cryptosporidium parvum antigens in human fecal specimens using the triage parasite panel enzyme immunoassay. J. Clin. Microbiol. 38, 9:333740.

Garcia, LS, 2016: Diagnostic Medical Parasitology. 6th Ed. ASM Press, Washington DC.

Gil, FF, Barros, MJ, Macedo, NA, Junior, CG, Redoan, R, et al, 2013: Prevalence of intestinal parasitism and associated symptomatology among hemodialysis patients. Rev. Inst. Med. Trop. Sao Paulo 55, 2:69-74.

Goni, P, Martin, B, Villacampa, M, Garcia, A, Seral, C, et al, 2012: Evaluation of an immune-chromatographic dip strip test for simultaneous detection of Cryptosporidium spp, Giardia duodenalis, and Entamoeba histolytica antigens in human fecal samples. Eur. J. Clin. Microbiol. Infect. Dis. 31, 8:2077-82.

Goodgame, RW, 1996: Understanding intestinal spore-forming protozoa: cryptosporidia, microsporidia, isospora, and cyclospora. Ann. Intern. Med. 124, 4:429-41.

Jones, WR, 1946: The experimental infection of rats with Entamoeba histolytica; with a method for evaluating the anti-amoebic properties of new compounds. Ann. Trop. Med. Parasitol. 40:130-40.

Kamki, Y, Singh, RH, Singh, NT, Lungram, P, Singh, BN, 2015: Intestinal protozoal and helminthic infections in immunocompromised patients attending RIMS Hospital, Imphal. J. Med. Soc. 29, 2:74-8.

Kucik, CJ, Martin, GL, Sortor, BV, 2004: Common intestinal parasites. Am. Fam. Physician 69, 5:1161-8.

Kukoschke, KG, Necker, A, Muller, HE, 
1990: Detection of Blastocystis hominis by direct microscopy and culture. Eur. J. Clin. Microbiol. Infect. Dis. 9, 4:305-7.

Kulik, RA, Falavigna, DL, Nishi, L, Araujo, SM, 2008: Blastocystis sp. and other intestinal parasites in hemodialysis patients. Braz. J. Infect. Dis. 12, 4:338-41.

Lallo, MA, Hirschfeld, MPM, 2012: Encephalito zoonosis in pharmacologically immunosuppressed mice. Exp. Parasitol. 131, 3:339-43.

Leelayoova, S, Taamasri, P, Rangsin, R, Naaglor, T, Thathaisong, $\mathrm{U}$, et al, 2002: In-vitro cultivation: A sensitive method for detecting Blastocystis hominis. Ann. Trop. Med. Parasitol. 96, 8:803-7.

Mahfouz, AA, El-Morshedy, H, Farghaly, A, Khalil, A, 1997: Ecological determinants of intestinal parasitic infections among pre-school children in an urban squatter settlement of Egypt. J. Trop. Pediatr. 43, 6:341-4.

McHardy, IH, Wu, M, Shimizu-Cohen, R, Couturier, MR, Humphries, RM, 2014: Detection of intestinal protozoa in the clinical laboratory. J. Clin. Microbiol. 52, 3:712-20.

Meijers, RW, Litjens, NH, de Wi, EA, Langerak, AW, van der Spek, A, et al, 2012: Uremia causes premature ageing of the $\mathrm{T}$ cell compartment in end-stage renal disease patients. Immun. Ageing 9, 1:19.

Shehata, A.I., Hassanein, F. \& Abdul-Ghani, R, 2019: Opportunistic parasitoses among Egyptian hemodialysis patients in relation to $\mathrm{CD} 4+$ T-cell counts: A comparative study. BMC Infect. Dis 19, 480. https://doi.org/10.1186/ s12879 $=019-4110-4$

Shetty, N, Prabhu, T, 1988: Evaluation of faecal preservation and staining methods in the diagnosis of acute amoebiasis and giardiasis. J. Clin. Pathol. 41, 6:694-9.
Shoaib, S, Hafiz, A, Tauheed, S, 2002: Role of trichrome staining techniques in the diagnosis of intestinal parasitic infections. J. Pak. Med. Assoc. 52, 4:152-4.

Stark, D, Barratt, JL, van Hal, S, Marriott, D, Harkness, J, et al, 2009: Clinical significance of enteric protozoa in the immunosuppressed human population. Clin. Microbiol. Rev. 22, 4:634-50.

Stensvold, CR, Suresh, GK, Tan, KS, Thompson, RC, Traub, RJ, et al, 2007: Terminology for Blastocystis subtypes--a consensus. Trends Parasitol. 23, 3:93-6.

Swierczewski B, Odundo E, Ndonye J, Kirera R, Odhiambo C, et al, 2012: Comparison of the triage micro parasite panel and microscopy for the detection of Entamoeba histolytical Entamoeba dispar, Giardia lamblia, and Cryptosporidium parvum in stool samples collected in Kenya. J. Trop. Med. 2012:564721.

Tan, ZN, Wong, WK, Nik, Z, Abdullah, B, Rahmah, N, et al, 2010: Identification of Entamoeba histolytica trophozoites in fresh stool sample: comparison of three staining techniques and study on the viability period of the trophozoites. Trop. Biomed. 27, 1:79-88.

Termmathurapoj, S, Leelayoova, S, Aimpun, P, Thathaisong, U, Nimmanon, T, et al, 2004: The usefulness of short-term in vitro cultivation for the detection and molecular study of Blastocystis hominis in stool specimens. Parasitol. Res. 93, 6:445-7.

Yakoob, J, Jafri, W, Beg, MA, Abbas, Z, Naz, S, et al, 2010: Irritable bowel syndrome: is it associated with genotypes of Blastocystis hominis. Parasitol. Res. 106, 5:1033-8.

Zierdt, CH, Rude, WS, Bull, BS, 1967: Protozoan characteristics of Blastocystis hominis. Am. J. Clin. Pathol. 48, 5:495-501.

\section{Explanation of figures}

Fig. 1: Parasites detected by microscopic examination: (A) B.hominis by Direct smear (40x) (B) B.hominis by Trichrome stain (100x); (C, D) Microsporidia by modified Trichrome stain (MTS) (100x).

Fig. 2: (A) Cryptosporidium by microscopic examination (MZN stain)(100x), (B) Cryptosporidium by ICT strip

Fig. 3: (A) G.lamblia by microscopic examination (iodine)(40x), (B) G.lamblia by ICT strip 

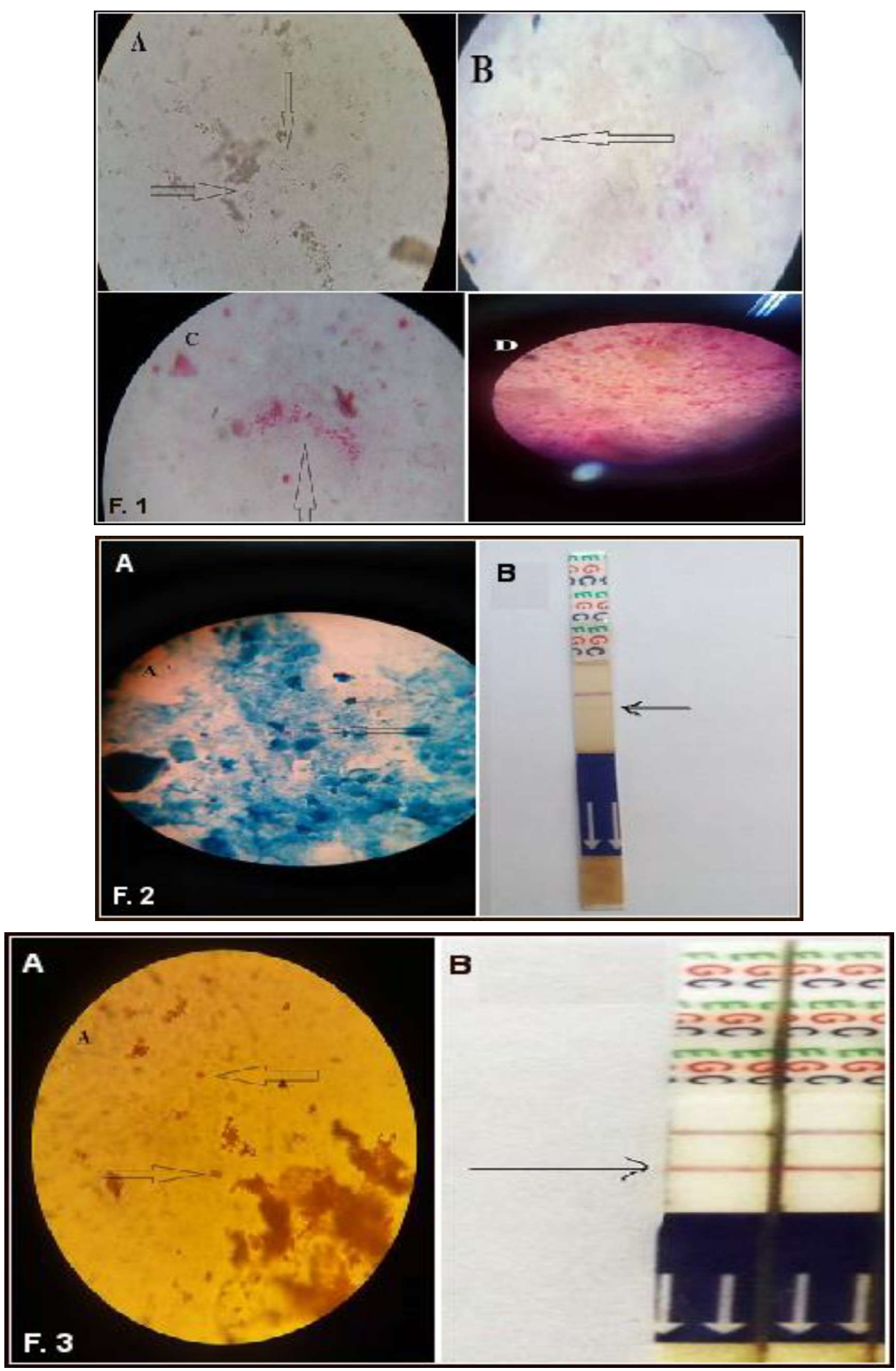